\title{
Eye Movement Detection Using EOG for Communication of People with Disabilities
}

\author{
Ryosuke Baba ${ }^{1}$, Takenao Sugi ${ }^{1}$, Yoshitaka Matsuda ${ }^{1}$, Satoru Goto ${ }^{1}$, Takao Yamasaki ${ }^{2}$, \\ Ayame Oishi ${ }^{2}$, and Naruto Egashira ${ }^{3}$ \\ ${ }^{1}$ Department of Advanced Technology Fusion, Saga University, \\ 1 Honjo-machi, Saga 840-8502, Japan \\ ${ }^{2}$ Department of Clinical Neurophysiology, Kyushu University, \\ 3-1-1 Maidashi Higashi-ku, Fukuoka 812-8582, Japan \\ ${ }^{3}$ National Institute of Technology, Kurume College, \\ 1-1-1 Komorino, Kurume, Fukuoka 830-8555, Japan \\ E-mail: sugi@cc.saga-u.ac.jp
}

\begin{abstract}
This research attempts the eye movement detection for the communication of people with disabilities by employing the electrooculographic (EOG) signals. Eye movements for the left and the right are detected by capturing the peak which appears in the waveform. Then, the judgment for left or right eye movement is conducted based on four parameters about the detected peak. To confirm the effectiveness of the eye movement detection method considered in this research, experiments were performed.
\end{abstract}

\section{Introduction}

Some people with disabilities have the difficulty in the communication using utterance, action, utilization of equipment etc. However, there is a possibilitity that his/her eye movement is available until he/she reaches totally locked-in state.

From this point of view, a nurse call system for people with such heavy disabilities has been developed by using image processing from camera images [1]. The system has a remarkable feature that non-contact detection of eye movement is realized. However, the success of detection is heavily affected by the condition of the environment (e.g., lighting condition). Furthermore, image processing may require relatively high computational burden. On the other hand, electrooculogram (EOG) [2] is recognized as one of the most useful information to capture the eye movements. Therefore, EOG signal may be effective in the extraction of human intention. Indeed, EOG signal was applied to the hands free remote operation of a mobile robot [3].
In this research, the detection of the eye movements for the communication of people with heavy disabilities is considered by using EOG signals.

\section{System Configuration}

The system configuration of this research is shown in Fig. 1. First, horizontal EOG is acquired from three electrodes placed on the subject's face as shown in Fig. 2. Exploring electrodes for EOG recording are placed at both left and right outer canthus. Secondly, acquired EOG is magnified by an $\mathrm{AC}$ amplifier with a high cutoff frequency of 15.9 [Hz] and a low cutoff frequency of $0.23[\mathrm{~Hz}]$ and are stored into the $\mathrm{PC}$ with the sampling interval of $5 \mathrm{~ms}$ via $\mathrm{AD}$ converter. Horizontal eye movement is extracted by signal processing.

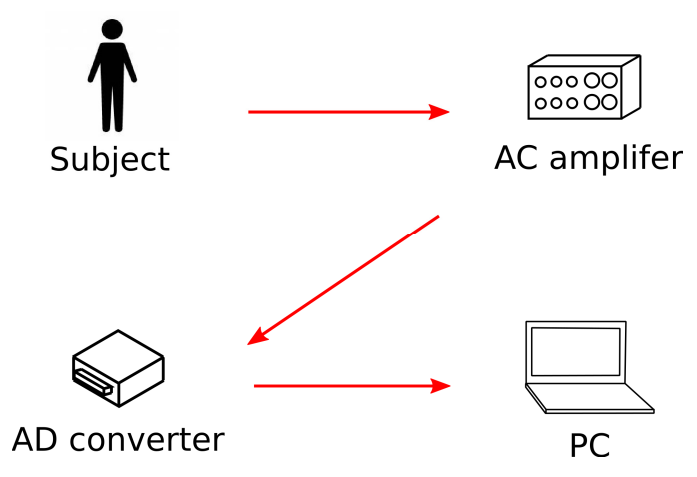

Fig. 1: System configuration 


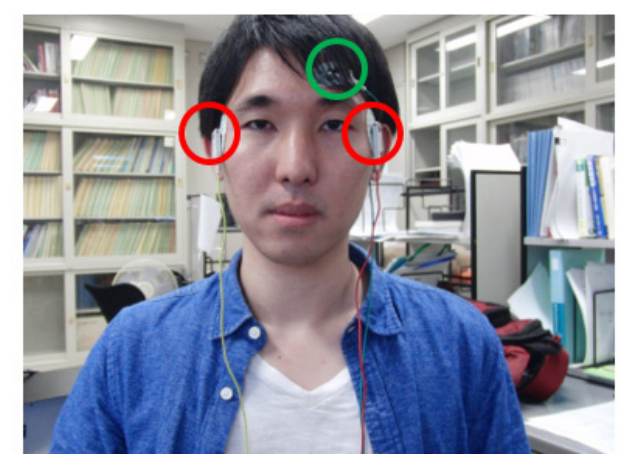

... Electrode to acquire EOG

... Electrode for ground

Fig. 2: Electrode placement

\section{Eye Movement Detection}

For the detection of eye movement, it is judged whether the user looks at the left or right from EOG signal. Figs. 3 and 4 show the relationship between EOG waveform and eye movement for the left and the right, respectively. As shown in Fig.3, rapid amplitude change occurs in the case that eyes move from front to left. Then after, the amplitude aradually decreases due to the filter property of $\mathrm{AC}$ amplifier. The case of gazing right direction shown in Fig.4 is vice versa. These figures indicate that the distinguishing peak corresponding to the direction of eye movement appears in the waveform. Therefore, in this research, eye movements are detected by extracting the peak of the signal $y(k)$.

\subsection{Peak Detection}

In order to extract the peak corresponding to eye movements, three points $\mathrm{F}, \mathrm{P}$ and $\mathrm{B}$ of the signal $y(k)$ shown in Fig. 5 or Fig. 6 are firstly detected [2]. Points $\mathrm{F}$ and $\mathrm{P}$ are defined by $y\left(k_{f}\right)$ and $y\left(k_{p}\right)$ satisfying

$$
\left[y\left(k_{*}+1\right)-y\left(k_{*}\right)\right]\left[y\left(k_{*}\right)-y\left(k_{*}-1\right)\right]<0
$$

where $y\left(k_{*}\right)$ means $y\left(k_{f}\right)$ or $y\left(k_{p}\right)$. (See Fig. 7.)

On the other hand, point B is defined by $y\left(k_{b}\right)\left(k_{b}>\right.$ $k_{p}$ ) satisfying

$$
y\left(k_{b}\right)<y\left(k_{f}\right) \quad \text { for left gaze }
$$

or

$$
y\left(k_{b}\right)>y\left(k_{f}\right) \text { for right gaze, }
$$

where minimal $k_{b}$ satisfying (2) or (3) is adopted. In the following argument, the times corresponding to $k_{f}$, $k_{p}$ and $k_{b}$ are expressed by $t_{f}, t_{p}$ and $t_{b}$, respectively.

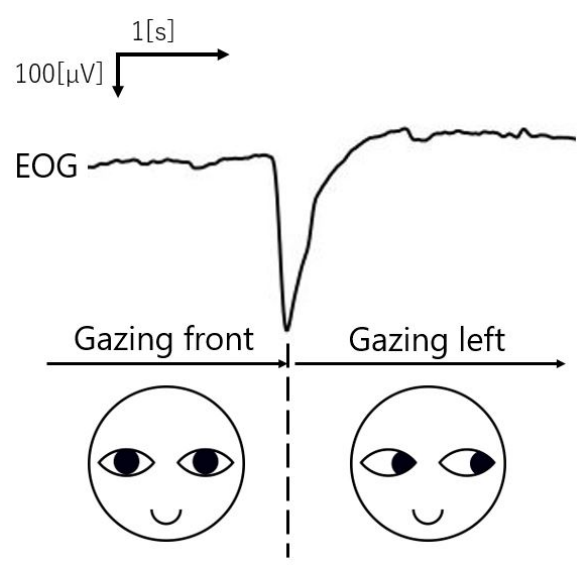

Fig. 3: Relationship between EOG waveform and eye movement for the left

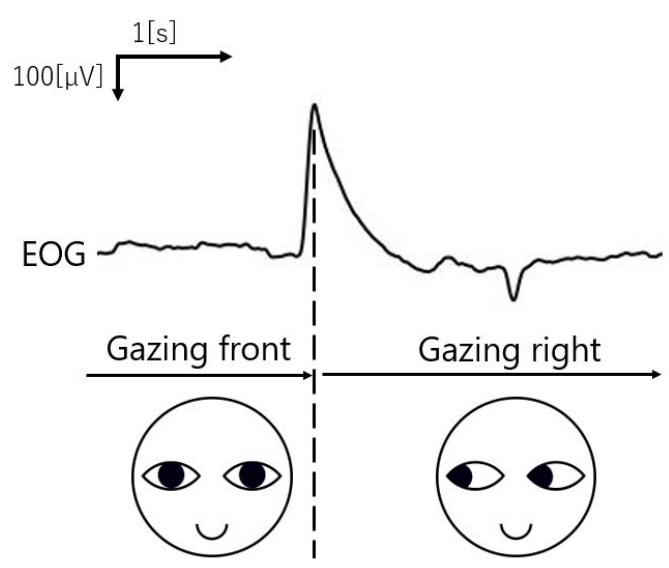

Fig. 4: Relationship between EOG waveform and eye movement for the right

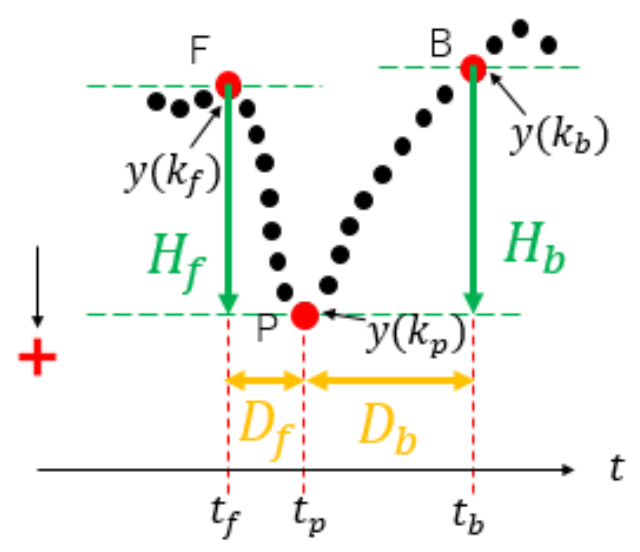

Fig. 5: Peak detection and judgment for left eye movement 


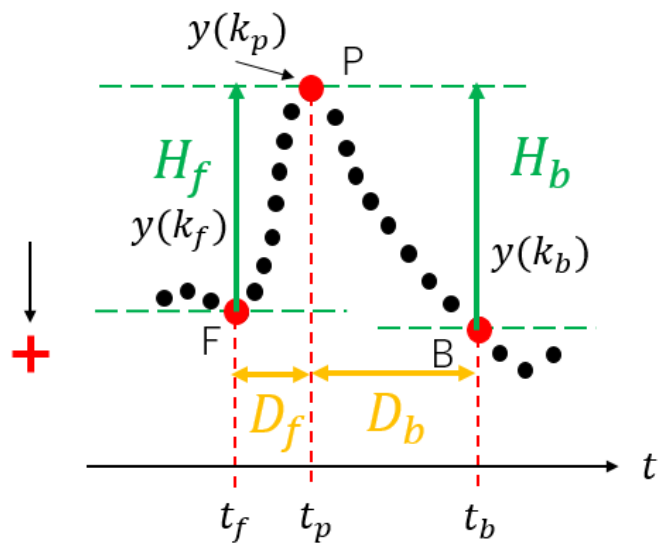

Fig. 6: Peak detection and judgment for right eye movement

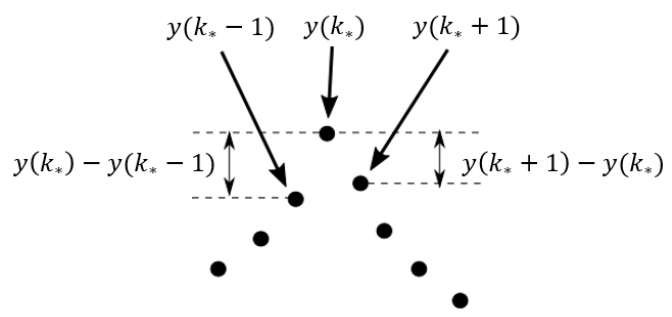

Fig. 7: Detection of points $\mathrm{F}$ and $\mathrm{B}$

\subsection{Judgment for Eye Movements}

Four parameters $H_{f}, H_{b}, D_{f}$ and $D_{b}$ shown in Figs. 5 and 6 are used for judging eye movements. $H_{f}$ and $H_{b}$ mean the amplitude and $D_{f}$ and $D_{b}$ correspond the duration of EOG waveform. Derivation of these parameters are listed in Table 1.

Table 1: Parameters for judgment

\begin{tabular}{|c|c|}
\hline Name & Parameter \\
\hline Forward amplitude & $H_{f}=y\left(k_{p}\right)-y\left(k_{f}\right)$ \\
\hline Backward amplitude & $H_{b}=y\left(k_{p}\right)-y\left(k_{b}\right)$ \\
\hline Forward duration & $D_{f}=t_{p}-t_{f}$ \\
\hline Backward duration & $D_{b}=t_{b}-t_{p}$ \\
\hline
\end{tabular}

\subsubsection{Judgment for Left Eye Movement}

If the following conditions:

$$
\begin{gathered}
H_{f} \geq \Lambda_{l h f} \\
H_{b} \geq \Lambda_{l h b} \\
\underline{\Lambda}_{l d} \leq D_{f}+D_{b} \leq \bar{\Lambda}_{l d} \\
H_{f} / D_{f} \geq \Lambda_{l s}
\end{gathered}
$$

are satisfied, then it is judged that there is a movement for left direction of the user's eyes. Thresholds for left judgment are listed in Table 2.

Table 2: Thresholds for left eye movement

\begin{tabular}{|c|c|}
\hline Name & Parameter \\
\hline Forward amplitude threshold & $\Lambda_{l h f}$ \\
\hline Backward amplitude threshold & $\Lambda_{l h b}$ \\
\hline Duration threshold (minimum) & $\underline{\Lambda}_{l d}$ \\
\hline Duration threshold (maximum) & $\bar{\Lambda}_{l d}$ \\
\hline Sharpness threshold & $\Lambda_{l s}$ \\
\hline
\end{tabular}

\subsubsection{Judgment for Right Eye Movement}

If the following conditions :

$$
\begin{gathered}
H_{f} \leq \Lambda_{r h f} \\
H_{b} \leq \Lambda_{r h b} \\
\underline{\Lambda}_{r d} \leq D_{f}+D_{b} \leq \bar{\Lambda}_{r d} \\
H_{f} / D_{f} \leq \Lambda_{r s}
\end{gathered}
$$

are satisfied, then it is judged that there is a movement for right direction of the user's eyes. Thresholds for right judgment are listed in Table 3.

Table 3: Right judgment parameter

\begin{tabular}{|c|c|}
\hline Name & Parameter \\
\hline Forward amplitude threshold & $\Lambda_{r h f}$ \\
\hline Backward amplitude threshold & $\Lambda_{r h b}$ \\
\hline Duration threshold (minimum) & $\underline{\Lambda}_{r d}$ \\
\hline Duration threshold (maximum) & $\bar{\Lambda}_{r d}$ \\
\hline Sharpness threshold & $\Lambda_{r s}$ \\
\hline
\end{tabular}

\section{Experiment}

In order to verify the effectiveness of the eye movement detection method developed in this research, experiments were conducted.

\subsection{Experimental Condition}

Three experiments were performed by three healthy subjects (Subject K, Subject N and Subject T). Experiments are done with that the subject lies down on the bed.

The subjects moved their eyes according to the instruction as listed in Table 4.

The thresholds for judgment were selected for all the subjects as shown in Table 5 . Those values were determined by using the data recorded from the subjects in advance. 
Table 4: Instruction for experiments

\begin{tabular}{|c|c|}
\hline Time $[\mathrm{s}]$ & Instruction \\
\hline 10 & Please move your eyes to the right \\
\hline 15 & Please return your eyes to the front \\
\hline 25 & Please move your eyes to the left \\
\hline 30 & Please return your eyes to the front \\
\hline 40 & Please move your eyes to the right \\
\hline 45 & Please return your eyes to the front \\
\hline 55 & Please move your eyes to the left \\
\hline 60 & Please return your eyes to the front \\
\hline
\end{tabular}

\subsection{Experimental Results}

Experimental results by Subject K, Subject N and Subject T are depicted in Figs. 8-10, respectively, where the detection results are also shown in the same figures. Furthermore, detection rate, undetected rate, over-detection rate and false detection rate are also s-
Table 5: Thresholds for experiments

\begin{tabular}{|c|c|}
\hline Parameter & Threshold \\
\hline$\Lambda_{l h f}$ & $250[\mu \mathrm{V}]$ \\
\hline$\Lambda_{l h b}$ & $250[\mu \mathrm{V}]$ \\
\hline$\underline{\Lambda}_{l d}$ & $0.3[\mathrm{~s}]$ \\
\hline $\bar{\Lambda}_{l d}$ & $1.5[\mathrm{~s}]$ \\
\hline$\Lambda_{l s}$ & $600[\mu \mathrm{V} / \mathrm{s}]$ \\
\hline$\Lambda_{r h f}$ & $-250[\mu \mathrm{V}]$ \\
\hline$\Lambda_{r h b}$ & $-250[\mu \mathrm{V}]$ \\
\hline$\underline{\Lambda}_{r d}$ & $0.3[\mathrm{~s}]$ \\
\hline $\bar{\Lambda}_{r d}$ & $1.5[\mathrm{~s}]$ \\
\hline$\Lambda_{r s}$ & $-600[\mu \mathrm{V} / \mathrm{s}]$ \\
\hline
\end{tabular}

ummarized in Table 6. From Figs. 8-10 and Table 6 we see that eye movements were perfectly detected in all experiments.

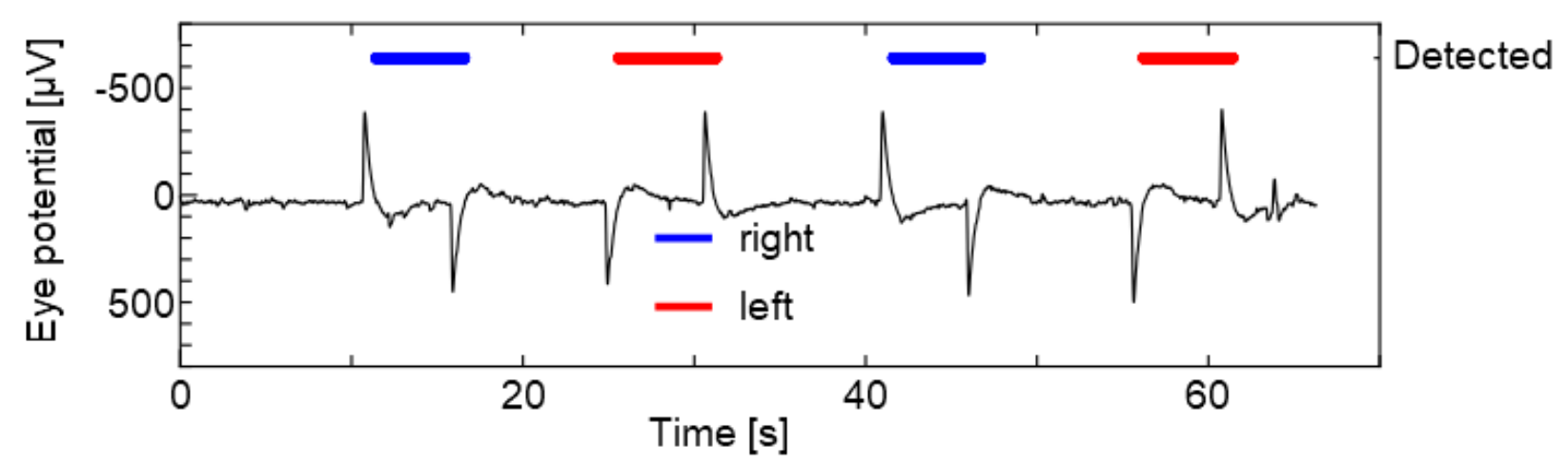

Fig. 8: Experimental result (Subject K)

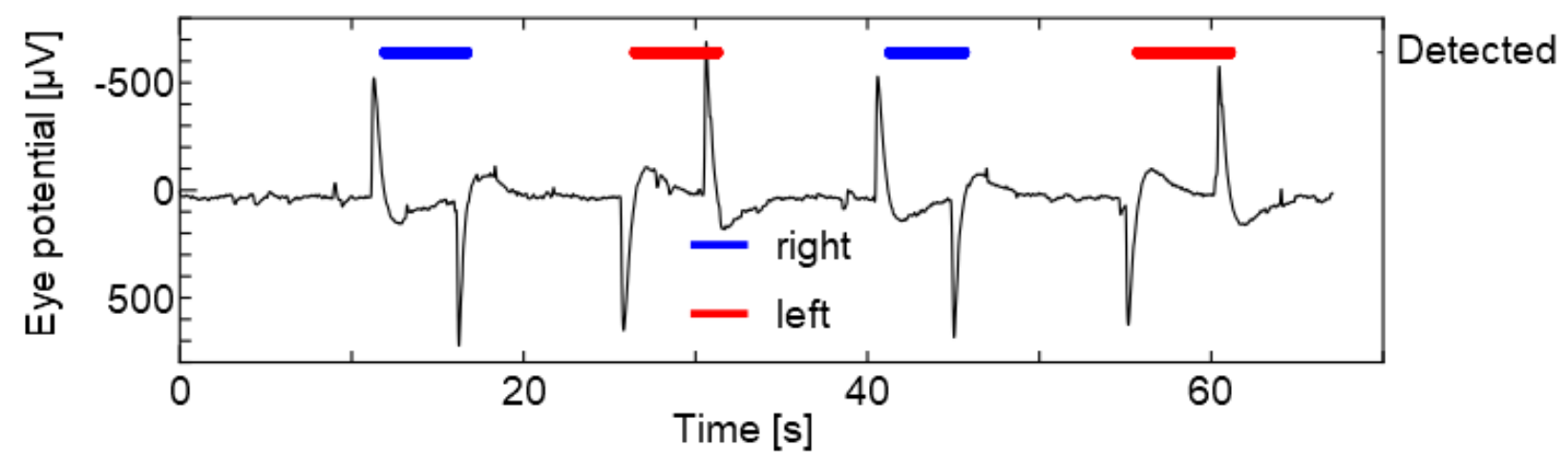

Fig. 9: Experimental result (Subject N) 


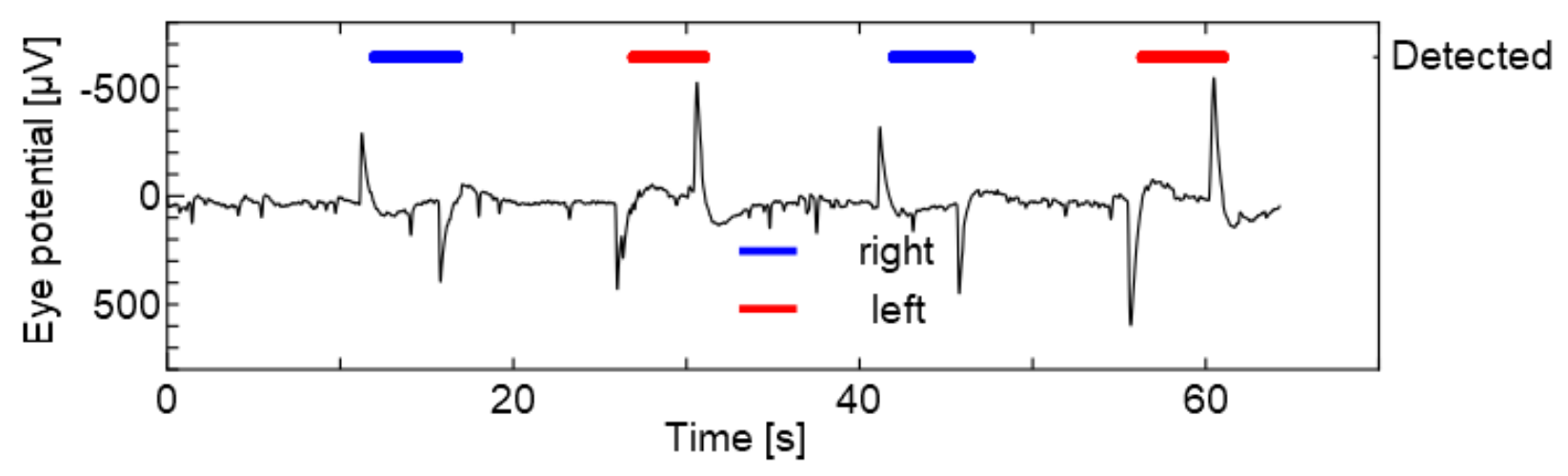

Fig. 10: Experimental result (Subject T)

Table 6: Detection results

\begin{tabular}{|c|c|c|c|}
\hline Subject & Subject K & Subject N & Subject T \\
\hline Detection rate [\%] & 100 & 100 & 100 \\
\hline Undetected rate [\%] & 0 & 0 & 0 \\
\hline Over-detection rate [\%] & 0 & 0 & 0 \\
\hline False detection rate [\%] & 0 & 0 & 0 \\
\hline
\end{tabular}

\section{Discussion}

In this research, the judgment of eye movements is realized by considering four parameters (i.e., forward amplitude $H_{f}$, backward amplitude $H_{b}$, forward duration $D_{f}$ and backward duration $D_{b}$ ) and suitable thresholds. Since the thresholds could be appropriately given for three subjects, sufficient accuracy of the eye movement detection was achieved. Therefore, the system developed in this research may also be effective for people with disabilities if suitable thresholds can be selected.

Furthermore, the experimental results imply that perfect manipulation of the method for communication by, at least, healthy users can be expected. Therefore, the method has an excellent potential to construct an effective communication system for people with disabilities.

\section{Conclusions}

In this research, the detection methodology for eye movement using EOG signal was investigated. Eye movements were detected by capturing the peak which appeared in moving user's eyes. Furthermore, it was verified that the developed detection system could achieve sufficient accuracy to apply to the construction of the communication system using EOG signal. In the future work, the authors will conduct some experiments of eye movement detection and communication system operation by people with disabilities.

\section{References}

[1] M. Minoda, T. Sugi, Y. Matsuda, S. Goto, T. Yamasaki and N. Egashira: Communication System by Using Eye Movement Extracted from Image Information, SICE Life Engineering Symposium 2016, Osaka International House Foundation, Osaka, Japan, 1C1-2, pp. 28-29 (in Japanese).

[2] T. Sugi, M. Nakamura, A. Ikeda, T. Nagamine and H. Shibasaki: Real-Time Processing of Blink Artifact Elimination on EEG Records by Use of the Normalized Averaging Method, Japanese Journal of Medical Electronics and Biological Engineering, Vol. 38, No. 1, pp. 33-41, 2000 (in Japanese).

[3] O. Yano, T. Sugi, Y. Matsuda, S. Goto, T. Yamasaki and N. Egashira: Single Channel Measurement of EOG and EMG Applicable for Hands Free Remote Operation System of a Mobile Robot, ICME International Conference on Complex Medical Engineering, OS19-5, pp. 94-95, Okayama, Japan, June 18-21, 2015. 